\title{
„ZEIGE MIR ALLE BILDER, AUF DENEN WÜRFEL ZU SEHEN SIND!“ DIE VERÖFFENTLICHUNG VON GRAFIKEN DES INSTITUTS FÜR SPIELFORSCHUNG IM REPOSITORIUM DER UB MOZARTEUM
}

\author{
von Rainer Buland \& Edith Leitner
}

Zusammenfassung: Rainer Buland (RB), Leiter des Instituts für Spielforschung und Edith Leitner (EL), Repositoriumsmanagerin der Universitätsbibliothek Mozarteum Salzburg, sprechen über die Entscheidung zur elektronischen Veröffentlichung von Grafiken im neu eingerichteten institutionellen Repositorium. Vor diesem innovativen Schritt waren administrative Hürden zu meistern und technische Anforderungen zu klären. Für Rainer Buland galt es zunächst, technische Standards wie Auflösung und Format zu definieren sowie Lizenzdetails festzulegen. Die Bibliothek war gezwungen, interessantes Neuland zu betreten, da für die Katalogisierung von Grafiken noch kein Regelwerk vorhanden ist. Das folgende Gespräch spiegelt die erfreuliche Zusammenarbeit von Forschung und Bibliothek bei der Erstellung des Grafik-Repositoriums wider.

Schlüsselwörter: Universität Mozarteum Salzburg; Institutionelles Repositorium; Spielforschung; Grafiken

\section{„SHOW ME ALL IMAGES, ON WHICH CUBES ARE DEPICTED!“ THE PUBLICATION OF GRAPHICS OF THE INSTITUTE FOR LUDOLOGY AND PLAYING ARTS IN THE REPOSITORY OF THE MOZARTEUM UNIVERSITY SALZBURG LIBRARY}

Abstract: Rainer Buland (RB), director of the Institute for Ludology and Edith Leitner (EL), Repository Manager of the Mozarteum University Library Salzburg, talk about the decision for the electronic publication of graphics in the newly established institutional repository. Before this innovative step administrative hurdles were to overcome and technical requirements to clarify. Rainer Buland had to define technical standards such as image resolution, format and license details. The library was forced to enter interesting new ground, because for the cataloging of graphics still no rules are available. The following conversation reflects the enjoyable cooperation of research and library in creating the graphics repository.

Keywords: Mozarteum University Salzburg; institutional repository; ludology; graphics

DOI: http://doi.org/10.31263/voebm.v71i1.1988

Dieses Werk ist lizenziert unter einer

Creative-Commons-Lizenz Namensnennung 4.0 International 


\section{Einleitung}

Das Institut für Spielforschung und Playing Arts an der Universität Mozarteum Salzburg befasst sich mit der Kulturgeschichte des Spiels und ist seit seiner Gründung 1990 ein Pionierunternehmen, das international vernetzt ist. Das Institut hat mit Hilfe von Sponsoren eine Forschungsbibliothek und Grafiksammlung aufgebaut, die als ein Teil der Universitätsbibliothek geführt wird. Neben Grafiken, auf denen Spielszenen zu sehen sind, zählen historische Spielbücher und Spielpläne zum Bestand dieser Fachbibliothek. Es ist heute wichtig, dass die Inhalte elektronisch zugänglich gemacht werden. Rainer Buland erklärte sich daher sofort bereit, am Aufbau des Repositoriums ${ }^{1}$ mitzuwirken. Das Repositorium wird von der OBVSG gemeinsam mit der Firma Walter Nagel/Semantics gehostet und beruht auf der proprietären Software Visual Library.

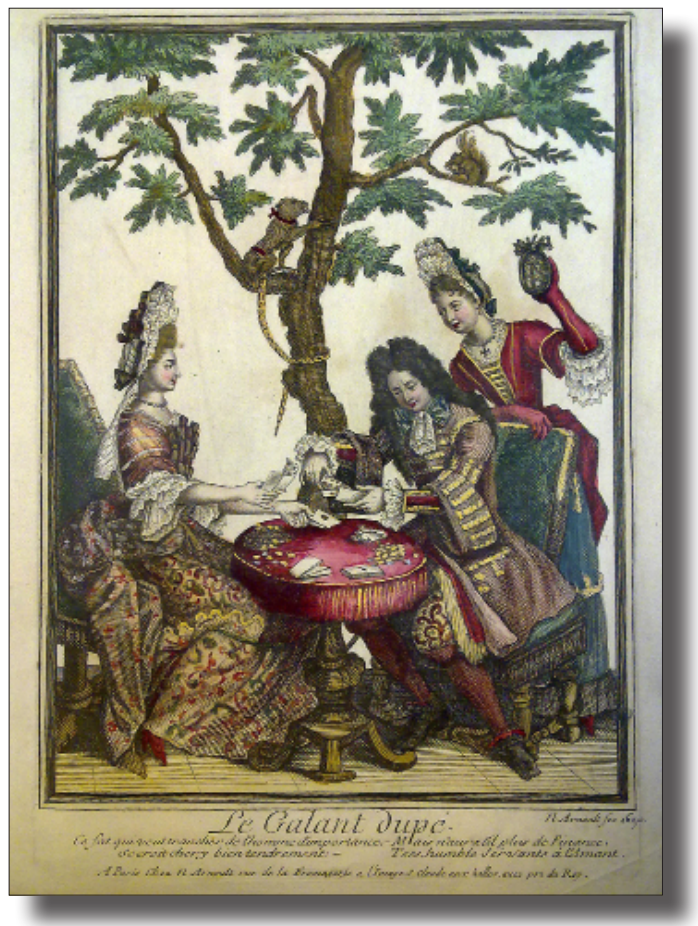

Abb. 1: Ein Stich von Arnoult, Paris 1690. Zwei Freundinnen nehmen einen „eitlen Gecken“, wie es in der Bildunterschrift heißt, mit etwas unlauteren Mitteln aus. Die eine steht hinter dem Adeligen und hält einen Handspiegel hoch, sodass ihre Freundin die Karten sieht. (Bild: Institut für Spielforschung CC-BY-NC) 
EL: „Le Galant dupé" ist Teil der Sammlung des Spielinstituts. In dieser Grafik aus 1690 wird gezeigt, wie zwei Freundinnen beim Kartenspiel einen Kavalier um sein Geld erleichtern. Können Sie ein paar Sätze über diesen Stich sagen, sozusagen als Beispiel für einen Eintrag ins Repositorium.

RB: Dieser Kupferstich ist ein gutes Beispiel, warum es so wichtig ist, dass die Forschung einen Beitrag zur inhaltlichen Entschlüsselung und damit zum Verständnis liefert. Es handelt sich nämlich um einen Modestich: Die Adeligen und das gehobene Bürgertum in Wien mussten wissen, was in Paris, vor allem in Versailles, der letzte Schrei war. Dazu wurden in Paris Puppen mit den neusten Kollektionen eingekleidet und in die wichtigsten Städte Europas transportiert. Dort konnten sich die Damen und Herren von Welt gegen ein kleines Eintrittsgeld ein Bild von der neusten Mode machen. Wer solche "Mode-Shows" nicht besuchen konnte, hatte die Möglichkeit, einen Modestich, wie eben den vorliegenden, zu kaufen. Die Kolorierung ist wunderschön und aufwändig gemacht, weil natürlich die Farben von entscheidender Bedeutung waren. Gleichzeitig wird hier aber auch eine Spielszene dargestellt: Zwei Freundinnen nehmen einen „eitlen Gecken“ aus. Dabei hilft ein Handspiegel, den eine Frau hinter dem Mann hochhält, sodass ihre Komplizin die Karten erkennen kann.

Es gibt dazu auch ein kleines fünf-minütiges Video auf YouTube ${ }^{2}$, in dem dieser Stich noch etwas ausführlicher erklärt wird.

EL: Man könnte dieses Bild einfach einscannen und auf einer beliebigen Website zugänglich machen. Warum soll „Der düpierte Galan“ in einem Repositorium auffindbar gemacht werden?

RB: Stellt man eine solche Grafik ohne ergänzende Ausführungen ins Netz, hätten Interessierte gar nicht die Möglichkeit, den Scan zu finden. Wenn wir nach Bildern suchen, müssen wir sprachliche Suchbegriffe verwenden. Im Repositorium sind die Bilder mit den Metadaten verknüpft. Erst damit lässt sich nach ihnen suchen. Gleichzeitig liefern die Metadaten Informationen zu Entstehung und Inhalt. Somit ist eine inhaltliche und wissenschaftliche Auseinandersetzung mit der Grafik möglich. Diese strukturellen Fragen sind aber Ihre Domäne.

EL: Die einheitliche Aufnahme von Metadaten zu Grafiken stellt die Bibliothek tatsächlich vor gewisse Herausforderungen. Schließlich existiert noch kein anerkanntes Regelwerk dazu. Ein solches entwickeln wir derzeit im Rahmen der AG-Bild. Das vorläufige Ergebnis wird am Deutschen Bibliothekarstag 2018 in Berlin präsentiert. 
Bis zur Finalisierung des Prozesses arbeiten wir noch mit einem Provisorium: einer internen, gemeinsam ausgearbeiteten Metadatenliste.

Aber kommen wir noch einmal zurück zum „Düpierten Galan“. Wie lässt sich die Zusammenarbeit von Forschung und Bibliothek darstellen?

RB: An diesem Beispiel lässt sich eine solche Kooperation wunderbar festmachen: Die Forschung liefert Informationen wie Datierung, die oft nur indirekt aus Papierqualität oder Kleidung der dargestellten Personen zu schließen sind. Weiters sind auch der genaue Name des dargestellten Spiels sowie Informationen zum Bildinhalt nur seitens der Wissenschaft zu leisten. Die Bibliothek verfügt über die erforderliche Kompetenz, diese Daten optimal aufzubereiten und zu standardisieren. Dazu gehört auch die Schreibweise des Namens eines Künstlers, ein großes Problem gerade in der Barockzeit, in der Namen nicht amtlich festgelegt waren wie heute, sondern dem Belieben des Schreibers folgten. Wir kennen das von Mozart, der zwar als Chrysostomos getauft wurde, sich immer Amadé nannte, der Nachwelt aber unter Amadeus bekannt ist.

EL: Diese Problematik wird in der Praxis über die Gemeinsame Normdatei (GND) gelöst, in der die wichtigsten Varianten so verknüpft sind, dass unterschiedliche Schreibweisen eines Namens in die Suchanfrage eingegeben werden können und dennoch zum richtigen Ergebnis führen.

Jetzt sollten wir vielleicht noch kurz darstellen, wie wir zu den Metadaten kommen.

RB: Zum Erstellen der Daten wird zunächst eine erste grobe Aufnahme von der wissenschaftlichen Hilfskraft am Spielinstitut gemacht: genaue Abschrift aller Angaben am Stich, Einfügen der Inventarnummer, Sprache, Land, genaue Abmessung und andere offensichtliche Angaben. Diese Liste ergänze ich mit Inhalten aus der Spielforschung. Das betrifft hauptsächlich die Inhaltsbeschreibung, Technik und Schlagwörter, wobei die Spielbezeichnungen nicht immer eindeutig sind, zumal wir auch fremdsprachige Bezeichnungen beibehalten. Diese Datenblätter gehen weiter an die Bibliothek.

EL: Hier werden sie ins Bibliothekssystem übertragen, also das Werk wird katalogisiert. Ich verknüpfe die Namen, wenn möglich, mit der GND, übersetze die Inhaltsangabe auf Englisch und ordne die Schlagwörter dem Klassifizierungssystem Iconclass zu. Im VL-Manager, der Bearbeitungsoberfläche des Repositoriums, lade ich den Scan der Grafik hoch. Da dieser im Dateinamen die AC-Nr. des Katalogisats führt, erfolgt eine automatische Verknüpfung damit. Im VL-Manager kann ich nun die Lizenzanga- 
be ergänzen. Sobald das Werk freigeben wird, ist es im Repositorium sichtbar. Über Nacht erhält es eine URN, einen permanenten, zitierbaren Identifier.

RB: Neben den Metadaten mussten wir zunächst für gute Scans der Grafiken sorgen. Wir haben am Institut einen ganz normalen Scanner, der den Zweck erfüllt. Das funktioniert grundsätzlich ganz gut. Größere Grafiken müssen jedoch in zwei Teilen gescannt und dann im Bildbearbeitungsprogramm zusammenfügt werden, was zusätzlichen Aufwand bedeutet. Noch größere Bilder fotografiere ich selbst, wozu das Institut eine kleine Studiobeleuchtung gekauft hat.

Die Grafiken werden für die Bereitstellung im Repositorium mit der höchstmöglichen Auflösungsstufe im JPG-Format eingescannt. Dieses Format kann mit einem Bildbearbeitungsprogramm wie GIMP sehr gut bearbeitet werden. Anschließend werden die Bilder auf die geeigneten Größen skaliert. Es hat beispielsweise bei einem Kupferstich wenig Sinn, wenn die Auflösung wesentlich größer ist als die einzelne Linie. Ein Kupferstich besteht einfach aus Linien, und mehr Bildinformation als diese Linie werde ich nicht bekommen. Anschließend kopiere ich das Logo in den Scan und wandle dies in ein PDF um.

EL: Die PDFs im Repositorium sind in einer so guten Auflösung, dass sie als Druckvorlage genommen werden können. Darüber haben wir lange diskutiert.

RB: Die Entscheidung dazu war nicht einfach. Einerseits sollten die Grafiken in guter Qualität der Forschung zur Verfügung stehen, andererseits fragten wir uns, ob eine Universität dazu da ist, kostenlose Druckvorlagen zu liefern. Wir haben uns letztlich jedoch dafür entschieden, die Bilder im Internet so hochauflösend zur Verfügung zu stellen, dass sie auch gedruckt werden können.

EL: Die PDFs können nun frei heruntergeladen und unter einer CC-BY-NC-Lizenz ${ }^{3}$, also nicht kommerziell, verwendet werden.

Zum Umgang mit den digitalisierten historischen Materialen gab es in der Bibliothek ebenfalls eine lange Diskussion. Wir haben uns im Sinne der Berliner Erklärung für eine CC-BY Lizenzierung entschieden.

Aber zurück zur Sammlung, zu den Grafiken des Instituts für Spielforschung: Wir haben bereits einen Stich kennengelernt. Können Sie noch generell etwas zur Sammlung sagen?

RB: Wir haben in unserer Fachbibliothek rund 3.000 Grafiken und 450 historische Bücher zur Kulturgeschichte des Spiels. Dies ist die weltweit größ- 
te Sammlung zu diesem Thema. Zeitlich umfasst sie die Jahre von 1505 bis 1920.

\section{EL: Warum gerade dieser Zeitraum?}

RB: Wir sammeln seit 25 Jahren, und in dieser Zeit waren leider keine handschriftlichen Originale aus dem Mittelalter zu erwerben. Unser ältestes Objekt ist ein Dürer-Stich von 1505. Die andere zeitliche Grenze hängt damit zusammen, dass die Photographie die Druckgrafik als Dokumentationsmedium ablöst. Die Druckgrafik wird ausschließlich zu einem künstlerischen Medium und löst sich von der Darstellung. Ein Stich aus dem 18. Jahrhundert hat - richtig interpretiert - einen dokumentarischen Wert. Also wenn darauf z. B. ein bestimmtes Kartenspiel dargestellt wird, dann kann ich daraus schließen, dass dieses Spiel in der Gesellschaft des Künstlers bekannt und wohl auch beliebt war. Oder denken wir an die unglaublich genauen und lebendigen Stiche aus den Zeitschriften des 19. Jahrhunderts. Sie geben keine realistische Sicht wieder, auch wenn die Kunstrichtung „Realismus“ heißt, sondern zeigen vielmehr eine genaue individuelle Sicht mit vielen Details. Die Künstlergrafik aus der Zeit nach dem Ersten Weltkrieg hat diesen dokumentarischen Wert nicht mehr, zumal sie zu einem guten Teil überhaupt abstrakt oder phantastisch ist. Deshalb hat es keinen wissenschaftlich-inhaltlichen Sinn, Spiel-Grafiken aus der Zeit nach dem Ersten Weltkrieg zu sammeln. Natürlich gibt es da auch die eine oder andere Ausnahme. Weil ich aber ungern „bis zum Ersten Weltkrieg“ sage, deshalb sage ich „,bis 1920“. Spielbücher übrigens sammeln wir bis heute. Da haben wir beispielsweise eine sehr interessante Unterkategorie "Spielbücher in der NS-Zeit", die noch einer wissenschaftlichen Aufarbeitung harrt.

\section{EL: Was kann man sich genau unter einer Spiel-Grafik vorstellen?}

RB: Grafik ist alles, was auf Papier gedruckt ist, also Flachware. Wir sammeln keine Spiele, keine Brettspiele in Schachteln, weil wir damit sofort ein gravierendes Raumproblem hätten. Natürlich mit Ausnahmen: Wir haben am Institut einige wenige besonders reizvolle oder pädagogisch interessante Brettspiele, einfach, um für Ausstellungen einige Beispiele zu haben. Spiel-Grafiken sind Spielpläne, die auf den Tisch gelegt wurden, um darauf zu spielen, wie beispielsweise das Eulenspiel oder das Gänsespiel. Beide sind seit dem 17. Jahrhundert überaus beliebt und oft aufgelegt. Vom Gänsespiel haben wir ungefähr zwanzig verschiedene Drucke vom 17. bis 
zum 19. Jahrhundert. Bei uns befinden sich auch viele Spiele, die heute völlig in Vergessenheit geraten sind, wie das im 19. Jahrhundert in Tirol weit verbreitete "Scarawanzl“. Da danke ich der Österreichischen Nationalbibliothek, dass sie einen großen Teil der Zeitschriftenbestände des 19. Jahrhunderts inhaltlich erschlossen und frei zugänglich online gestellt hat. Sonst wäre ich niemals auf die Erwähnung des Scarawanzl in einer Berliner Zeitung gestoßen. Ein Journalist, von dem ich nur die Initialen kenne „P.v.S.“, urlaubte im Sommer im Tiroler Achental. Als er wieder in Berlin war, veröffentlichte er ab Herbst 1865 in der Wochenzeitung „Morgenblatt für gebildete Leser" eine Artikelserie über seine Erlebnisse in Tirol. Er beschrieb darin auch, wie abends das Scarawanzl vor der Hütte auf den Tisch gelegt und eifrig gespielt wurde. Es ist eine Variation des Poch-Spiels, ein Glücksspiel.

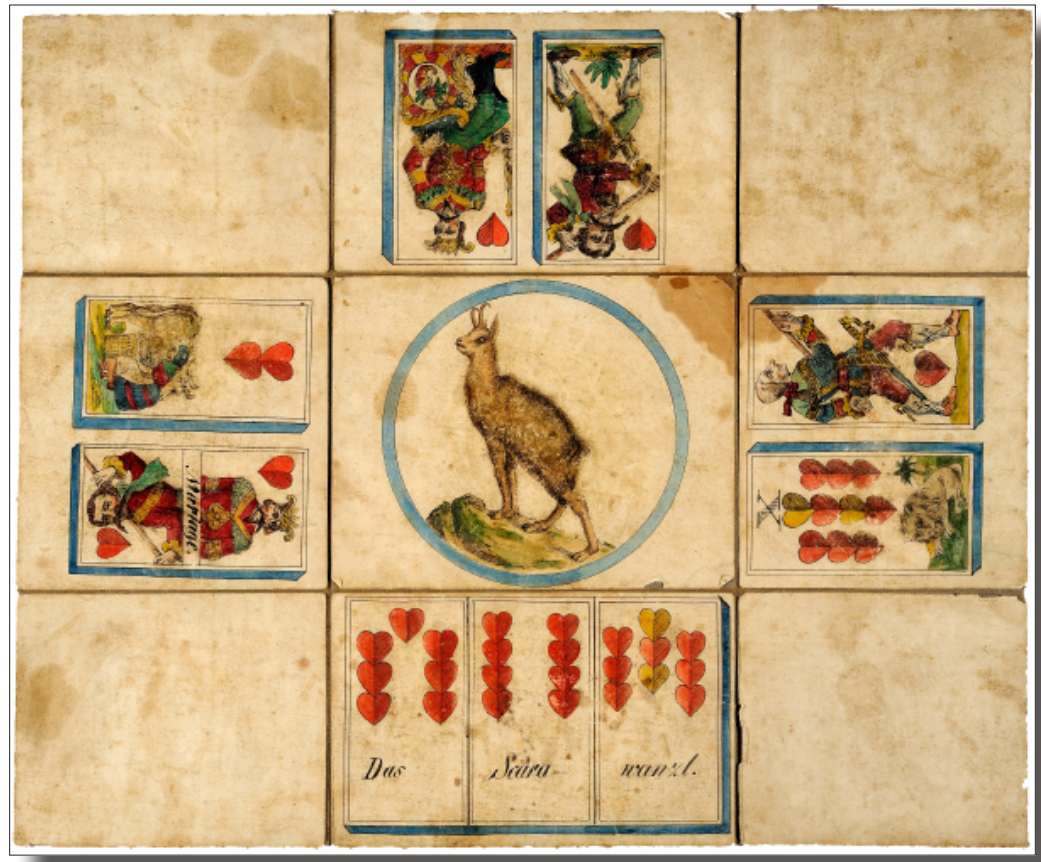

Abb. 2: „Scarawanzl“, eine Variante des Poch-Spieles, das im 19. Jahrhundert nachweislich in Tirol gespielt worden ist. (Bild: Institut für Spielforschung CC-BY-NC)

Der größte Teil der Grafiken gibt jedoch Spielszenen wieder: Spielgesellschaften beim Kartenspiel, beim Würfeln, bei Sportspielen und so weiter. Einen besonderen Schwerpunkt habe ich in den letzten Jahren auf den Be- 
reich „Musik und Spiel“ gelegt, also Stiche, auf denen man sieht, wie musiziert wird. Letztlich ist Musik-Machen auch ein Spiel.

Wir können die ganze Kulturgeschichte des Spiels seit der Renaissance aus eigenen Beständen bebildern. Das ist weltweit einmalig. Deshalb wurde das Institut auch in die Liste der nationalen Großforschungs-Infrastruktur aufgenommen, was eine große Auszeichnung ist und zeigt, dass der Wert der Sammlung durchaus gesehen wird.

\section{EL: Wie genau werden diese Objekte nun erforscht? Lässt sich dies an einem Beispiel demonstrieren?}

RB: Ich habe 2015 ein Gänsespiel für das Institut ersteigert, das im Auktionskatalog mit „aus dem 16. Jahrhundert" beschrieben war.

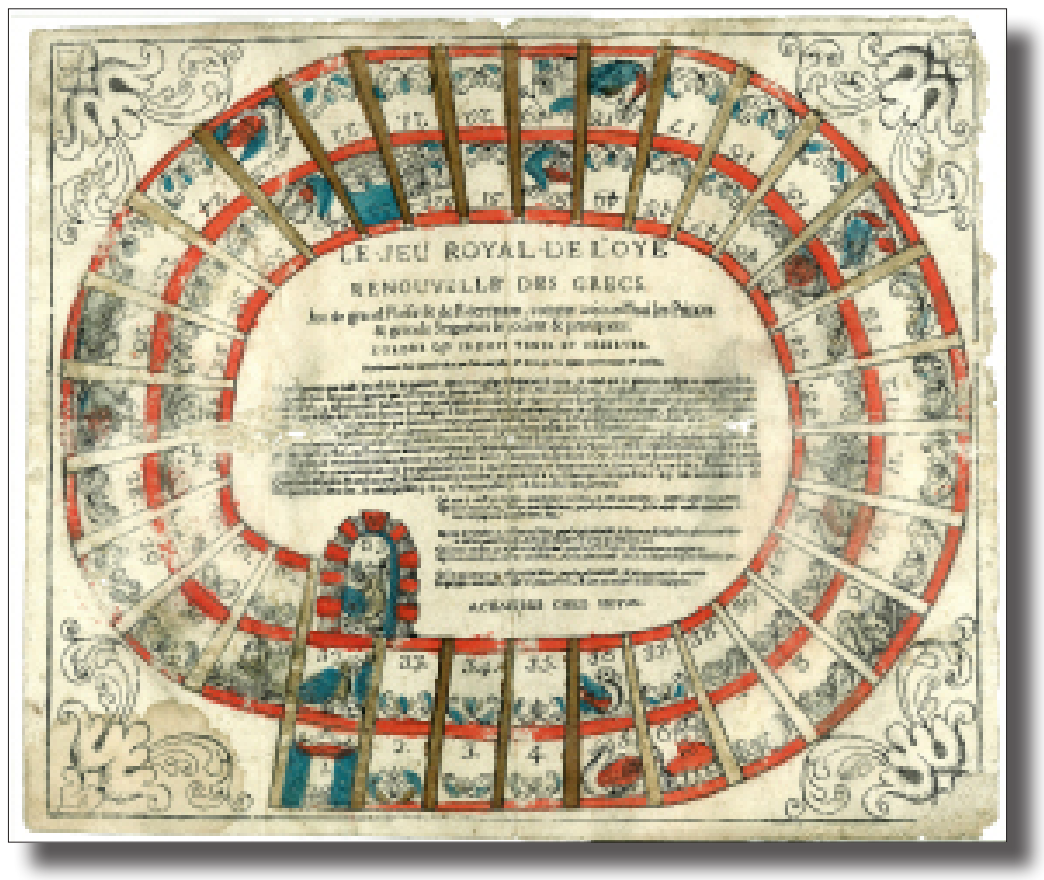

Abb. 3: „Le jeu royale de I'oye“, ein sehr seltenes Gänsespiel, das im 18. Jahrhundert in Chartres gedruckt worden ist. (Bild: Institut für Spielforschung CC-BY-NC)

Auf dem Spielplan findet sich der Hinweis, dass es bei „Hoyau“ in Chartres gedruckt worden ist. Ich habe nachgeforscht. Es hat tatsächlich einen Stecher dieses Namens in Chartres im 16. Jahrhundert gegeben, nämlich 
Germain Hoyau, der von 1508 bis 1580 lebte. Allerdings arbeitete zwei Jahrhunderte später zwischen 1751 und 1776 auch ein Drucker dieses Namens, Pierre Hoyau. Welcher der beiden hat das Spiel tatsächlich gedruckt? Durch den Vergleich der Schrift mit anderen Werken der beiden konnte ich nachweisen, dass das Gänsespiel von Pierre Hoyau und somit aus dem 18. Jahrhundert stammt.

\section{EL: Es ist also jünger als im Auktionskatalog angegeben - eine Enttäuschung?}

RB: Ja, es war eine gewisse Enttäuschung, aber ich habe die Angabe „16. Jahrhundert" ohnehin nie geglaubt. Das wäre eine echte Sensation gewesen, weil es aus dieser Zeit keine eindeutig datierbaren Gänsespiele gibt.

EL: Jetzt sollten wir noch über eine spezielle Möglichkeit des Repositoriums sprechen, nämlich die Vernetzung der Datensätze.

RB: Deswegen machen wir ja den Aufwand, die Grafiken in den Bibliotheksverbund einzupflegen und nicht z. B. auf eine eigene Website zu stellen. Wenn ich in Zukunft ein Stichwort eingebe, z.B. Tarock, dann bekomme ich die Grafiken, auf denen wir sehen, wie die Leute tarockieren. Aber ich bekomme auch die Tarock-Bücher und kann die Spielregel oder die Geschichte des Spiels nachlesen. Inwieweit wir in Zukunft die Daten direkt verlinken, ist eine Ressourcenfrage, z.B. ließe sich ein Stich wie „Le Galant dupé" mit einem historischen Spielbuch verlinken, in dem das Kartenspiel erklärt wird, oder mit Sekundärliteratur dazu. Das haben wir noch gar nicht umfassend diskutiert.

EL: Noch eine Frage, die uns wahrscheinlich viel Arbeit machen wird: Sie haben ja schon die Brettspiele erwähnt, die zwar nicht im Zentrum des Sammelinteresses stehen, aber letztlich doch zur Sammlung gehören. Wie werden die in das Repositorium eingearbeitet werden?

RB: Ja (lacht), die werden uns noch einiges Kopfzerbrechen bereiten. Es sind ungefähr vierzig Brettspiele vom 18. Jahrhundert bis zum Zweiten Weltkrieg und ungefähr ebenso viele Spielkarten. Das wird eine Herausforderung der Katalogisierung: kein Autor, kein Künstler, kein Datum, verschiedene Materialien, Spielfiguren aus Zinn, Würfel aus Bein. Wenn wir den Verlag ermitteln können, müssen wir schon froh sein. Aber: Wie werden sich die fehlenden Metadaten mit dem Anspruch eines Repositoriums vertragen? 
EL: Das hört sich tatsächlich nach einer noch größeren Herausforderung für uns beide an, als es die Grafiken sind. Aber sobald diese offenen Punkte geklärt sind, ergibt das sicherlich eine weitere einzigartige digitale Sammlung.

Mir ist noch eine wichtige Diskussion beim Aufbau des Repositoriums in Erinnerung: Wir waren eigentlich bereits im Endstadium, da haben Sie die Frage nach der Sprache des Repositoriums bzw. der Metadaten gestellt.

RB: Das war eine plötzliche Idee. Wir gehen ganz selbstverständlich immer vom Deutschen aus. Gerade die Grafiken sind aber für die internationale Forschergemeinschaft von Bedeutung - und diese spricht Englisch. International gesehen spricht kaum ein Spielforscher Deutsch. So gesehen bin ich wirklich sehr froh, dass mein spät eingebrachter Vorschlag der Zweisprachigkeit, Deutsch und Englisch, noch aufgenommen worden ist. Damit haben wir eine internationale Lösung, die langfristig Bestand haben wird.

Apropos langfristiger Bestand. Die Grafiken sind in einem feuerfesten Safe unter säurefreiem Museumspapier gut gelagert. Wie sieht es mit der Haltbarkeit der elektronischen Daten aus, die wir produzieren?

EL: Das berührt einen heiklen Punkt. Derzeit garantiert uns die Firma, die die Software für unser Repositorium entwickelt hat, eine dauerhafte Archivierung von zehn Jahren. Eine Lösung für eine tatsächliche Langzeitarchivierung über diesen Zeitraum hinaus ist generell noch nicht gefunden worden. Die Forschung ist jedoch zuversichtlich. Wir hoffen, dass mit den gewählten Formaten und den standardisierten Metadaten von unserer Seite die Grundlage geschaffen wird, die Daten in Zukunft verwenden zu können. Trotz der noch ungelösten Problematik einer Langzeitarchivierung sehe ich aber die Vorteile eines Repositoriums überwiegen, beispielsweise eine weltweite Sichtbarkeit und Zugriffsmöglichkeit rund um die Uhr.

RB: Apropos Zukunft: Vielleicht wird in nicht allzu ferner Zeit die Künstliche Intelligenz das Internet nach Bildmustern durchsuchen können. Ich sage dann beispielsweise zu meinem Suchroboter: „Zeige mir alle Bilder, auf denen Würfel zu sehen sind!“ Die KI weiß, wie ein Würfel aussieht und zwar von allen Perspektiven, durchsucht in der Folge alle Bildinhalte nach diesen Mustern und liefert mir eine Trefferliste. Aber selbst wenn dies einmal möglich sein sollte, blieben die Treffer für die Forschung unbefriedigend: Erstens sind auch alle Schachteln und Kisten dabei, die zufällig kubisch sind und einen Punkt tragen. Zweitens sind damit noch immer viele Fragen nicht beantwortet: der Name des Künstlers/der Künstlerin, Datierung, Drucktechnik oder inhaltliche Beschreibungen. Wir müssten also doch wieder auf die von uns eingepflegten Metadaten zurückgreifen. 
EL: Darin sehe ich auch den großen Wert des Repositoriums: eine frei zugängliche Sammlung, die als Art Galerie mit allen Informationen zur Verfügung steht.

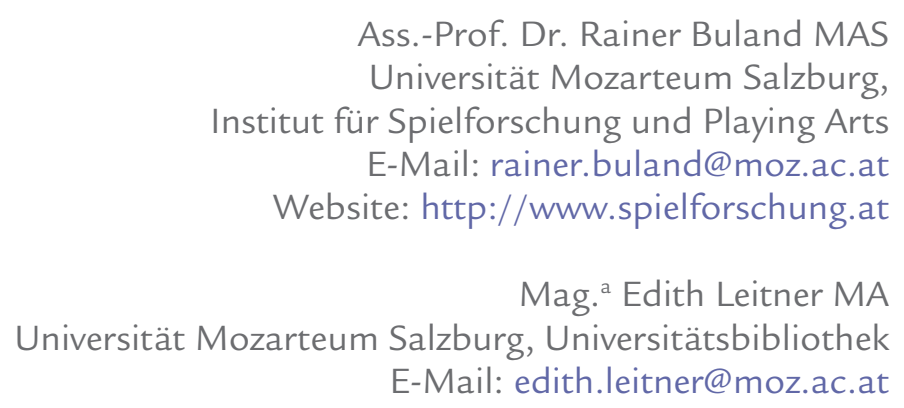

1 Die ersten Objekte sind im Repositorium unter http://repository.moz. ac.at zu finden.

2 Rainer Buland: Arnoult Galant dupé 1690, Youtube: https://youtu.be/ EZv3OyC04iY

3 https://creativecommons.org/licenses/?lang=de 